\title{
A Realistic Approach to Calculate VaR
}

\author{
Liangxin Li \\ Department of Finance, Hunan International Economics University \\ Changsha 410205, China \\ E-mail: apolo1@sina.com
}

\begin{abstract}
Value at Risk (VaR) has become the standard measure of market risk employed by financial industry for both internal and regulatory purposes. VaR is defined as the value that a portfolio will lose with a given probability, over a certain time horizon (usually one or ten days). Despite its conceptual simplicity, its measurement is still a very challenging statistical problem and none of the methodologies developed so far give satisfactory solutions. In this paper, we develop a new approach by expanding the realistic return distribution as linear summation of the standard normal distribution function with its coefficients as Legendre polynomial series to improve the calculation of VaR. One can obtain the distribution function toward the realistic distribution in any assumed precision. This approach outcomes the usual VaR calculation by assuming the normal distributions.
\end{abstract}

Finally, we test our approach through a real world data. It is found that our approach give more accurate results for the $\mathrm{VaR}$ and also more accurate distribution function than the usual normal distributions.

Keywords: VaR, Risk, Distribution, Legendre, Polynomial

\section{Introduction}

Financial disasters like Enron and Wall Street accounting fraud have emphasized the importance of effective risk management for financial industry. The use of quantitative risk measures has become an essential management tool to be placed in parallel with the models of returns. These measures are used for investment decisions, supervisory decisions, risk capital allocation, external regulation and efficient bankoperations(Markowitz,1952,pp79-91;Eagle,1999,P7341;Beder,,1995,pp12-24;Boudoukh,etal,1998,pp64-67;Foresi, etal,1995,pp1749-1778).

VaR is an estimate of how much a certain portfolio can lose within a given time period and at a given confidence level. Despite its simplicity conceptually, the measurement of VaR is still a very challenging statistical problem and none of the methodologies developed so far gives satisfactory solutions. The major challenge comes from two aspects: first, we need to know the accurate distribution function; second, we need to know how the future distribution will evolve. In this paper, we will focus on this first issue. For the second issue, there are many approaches such as time series, conditional entropy, random matrix (Mehta,1995; Granger,etal,1989,pp87-96; Hong,1999,pp1201-1220; Georges, etal, 2000, pp429-439) to improve the forecast of future distribution functions.

In the literature, VaR calculations usually assuming a simple Normal distribution function to do the calculations. Although it gives estimation on $\mathrm{VaR}$, it is too simple to include more information on the distortion from the normal distribution and will affect the precision of the VaR calculation.

In order to improve the analysis, we propose a Legendre polynomial expansion as follows:

$$
f(x)=\left(a_{0} P_{0}(x)+a_{1} P_{1}(x)+\ldots . .\right) \phi(x)
$$

where Ps are Legendre polynomials as in Appendix A. $\phi$ is the normal distribution. Along this line, there is so-called Gram-Charlier expansion with the coefficients as the Hermite Polynomials (Jarrow,etal,1982,347-369; Jondeau,etal,2001,pp1457-1483; Parton,etal,1952,pp425-427 and Appendix B)which causes negative probability and normalization problem for our data.

By expanding the realistic distribution in a Legendre Polynomials, we expect this approach will give us more accurate results for VaR calculations.

\section{Theoretical Model of VaR}

\subsection{Value at Risk models}

The existing models for calculating VaR differ in the methodology they use, the assumptions they make and the way they are implemented. However, all the existing models follow a common general structure, which can be summarized in three points:1) the portfolio is marking-to-market daily; 2)the distribution of the portfolio's returns is estimated; 3)the VaR of the portfolio is computed. 
Beder(1995,pp.12-24) applies eight common VaR methodologies to three hypothetical portfolios. The results show the differences among these methods can be very large, with VaR estimates varying by more than 14 times for the same portfolio. Clearly, there is a need for a statistical approach to estimation and model selection.

\subsection{Our model}

Our model focuses on the improvement of the estimation of the real distribution function from the past return data.

We expand the real distribution function $\mathrm{f}(\mathrm{x})$ as the Legendre polynomial as in Eq.1

$$
f(x)=\left(a_{0} P_{0}(x)+a_{1} P_{1}(x)+\ldots . .\right) \phi(x)
$$

They are orthogonal with each other in the domain $[-1,1]$, i.e.:

$$
\int_{-1}^{1} P_{i}(x) P_{j}(x) d x=\frac{2}{2 i+1} \delta_{i, j}
$$

We can scale the Legendre domain into any domain [-A, A]. Considering the decay rate of exponential function of the normal distribution, we calculate the a's as in Eq.(1.4A) in the Appendix A:

$\int d x f(x) / \phi(x) P_{i}(x)=a_{i} N_{i}=<f(x) / \phi(x), P_{i}(x)>$

With $\mathrm{Ni}=2 / 2 \mathrm{i}+1$. In real

data analysis, we do it by summation over the data return.

Now, Calculate VaR:

$$
G(A)=\int_{A}^{\infty} f(x) d x=\left.F(x)\right|_{A} ^{\infty} \geq \alpha
$$

Here, $\left.F(x)\right|_{A} ^{\infty}$ can be integrated

analytically as in the appendix A. Then, we have

$\operatorname{VaR}_{\alpha}=A=G^{-1}(\alpha)$.

This is the modified VaR.

In the practical calculation, we compute the VaR through the plot of the curves: $\left\{\begin{array}{l}y=G(x) \\ y=\alpha\end{array}\right.$, the intersection of these two curves is the modified VaR as shown in Fig.1:

$\operatorname{VaR}_{\alpha}=A=G^{-1}(\alpha)$.

Insert Figure 1 Here

\section{Real data analysis}

As a test of our approach above, we pick the index data from Chinese Shanghai stocks exchange index as the real data. We analyze it as three steps: 1) we first calculate the VaR for usual assumption of normal distribution, 2) then calculate VaR from our modified distribution. Finally, 3) we compare the model results with the direct calculation of VaR from the real data. Furthermore, we plot the distribution function for both normal distribution and modified distributions and compare with the real data distribution.

\subsection{The VaR calculations}

In order to facilitate the calculation, we take $\alpha=95 \%$. i.e., the probability of $95 \%$ to lose the VaR in a month: $\mathrm{N}=30$ days. For normal distribution, $\operatorname{VaR}=1.65 \mathrm{~N} \sigma$. We take $r_{t}=\ln \left(I_{t} / I_{t-1}\right)$ as the t-day's daily return of the index. The period is from 1992-1-2 to 2000-12-29 for 9 years.

For comparison, we calculate the Legendre polynomial results as in Eq. 1 to $5^{\text {th }}$ order:

$$
f(x)=\phi(x)\left(-1.551-2.980 P_{1}(x)-1.086 P_{2}(x)+2.989 P_{3}(x)+5.974 P_{4}(x)+4.666 P_{5}(x)\right)
$$


We plot the results as the way shown in Fig.1.

Let $\mathrm{G}(\mathrm{x})=0.95$, we can solve $\mathrm{x}$ for VaR. The results are shown in the following table:

Insert Table 1 Here

From table 1, we find that the first order distribution is good enough to catch the real VaR. The normal distribution is much off both in VaR and the distribution as shown in the following. The higher order correction doesn't improve the VaR but really catch the peak shape. So the tail seems not get the weight in the expansion.

\subsection{Plots of the distribution functions}

As shown in Fig.2, the standard normal distribution seems far off from the real data distribution which got a much sharp peak. The first Legendre correction doesn't change the normal distribution much in the peak but shift the VaR toward the real VaR closer. It should be a successful try toward the real VaR. The second and the third Legendre corrections are closer to the real data distribution in the peak, but the VaR is little off the real VaR. It catches the peak shape but ignores the tail. We also notice that peaks shift little away from the normal peak. Especially, the third Legendre correction shows negative probability in the near-peak region which is a sign the real distribution is far off the normal distribution, and certainly the VaR is far off the real one. The Higher order correction should be careful to use due to the stability of the coefficients calculations. We present the VaRs in the table 1. We conclude that our Legendre expansion is a good attempt to get a better VaR toward the realistic data. But our real data is far off from the normal distribution so that our expansion to the $5^{\text {th }}$ order is not enough.

Insert Figure 2 Here

\section{Conclusion}

We proposed a new way to calculate VaR. Any real distribution functions are expanded into a combination of the standard normal distributions with its coefficients as the Legendre polynomials. To get more accurate distributions, we need more information from the stock return than just the average return and standard deviation. Unlike most of the models assuming the normal distribution, we think the normal distribution only catches two pieces of information from the stock's returns. We approach real world stock's return's distributions by expanding the real distributions as Legendre polynomials of normal distribution's. We analyze a real stocks data and find that our approach present systematically more accurate distribution and of course more precise VaR as expected.

\section{Appendix}

\section{Appendix A: Legendre Polynomial Expansions:}

The Legendre polynomial is defined as:

$$
\begin{aligned}
& P_{0}=1, \\
& P_{1}=x \\
& P_{2}=\frac{3 x^{2}-1}{2} \\
& P_{3}=\left(5 x^{3}-3 x\right) / 2 \\
& P_{4}=\frac{35 x^{4}-30 x^{2}+3}{8}, \\
& \ldots \ldots \ldots . \\
& P_{i}(x)=\frac{(2 i-1) P_{i-1}(x)-(i-1) P_{i-2}(x)}{i}, i=2,3, \ldots \ldots
\end{aligned}
$$

These bases are orthogonal with each other in the domain $[-1,1]$, i.e.:

$$
\int_{-1}^{1} P_{i}(x) P_{j}(x) d x=\frac{2}{2 i+1} \delta_{i, j}
$$

The amplitude of the LPs are within $[-1,1]$ for the domain $[-1,1]$. Since the Normal distribution are strong localized around 0 , so, we can scale the LPs to any region which is suitable to the real data's distribution, such as $\mathrm{x}=\mathrm{y} / \mathrm{L}$, $\mathrm{y}$ belongs to $[-\mathrm{L}, \mathrm{L}]$. As long as after $\mathrm{L}$, the real data $\mathrm{f}(\mathrm{x})$ equals zero, then we can virtually expand the $\mathrm{f}(\mathrm{x})$ in the region $[-\infty, \infty]$, as 


$$
f(x)=\left(a_{0} P_{0}(x)+a_{1} P_{1}(x)+\ldots . .\right) \phi(x)
$$

The integral can be done analytically as follows:

$$
\int_{-\infty}^{\infty} f(x) d x=a_{0}+a_{1}<x>+a_{2}\left(3<x^{2}>-1\right) / 2+\ldots
$$

where $<x>$ is the average over normal distribution $\mathrm{N}(0,1)$. etc. We only need to calculate the normal moments to get the normalization. The coefficients can be calculated from the orthogonalization of P's:

$$
\int d x f(x) / \phi(x) P_{i}(x)=a_{i} N_{i}=<f(x) / \phi(x), P_{i}(x)>
$$

with

$$
N_{i}=2 /(2 i+1)
$$

\section{Appendix B: Hermite Polynomial Expansions}

First, to any distribution function, we expand as following:

$$
f_{X}=c_{0} \varphi(x)+c_{1} \varphi^{\prime}(x)+\frac{c_{2}}{2 !} \varphi^{\prime \prime}(x)+\frac{c_{3}}{3 !} \varphi^{(3)}(x)+\cdots \cdots
$$

Where $c_{i}$ as the expanding coefficients to be determined from the data, $\varphi(x)$ is the standard normal distribution with average 0 and standard deviation $1 . N(0,1)$, i.e., the density function is:

$$
\varphi(x)=\frac{1}{\sqrt{2 \pi}} \exp \left(-\frac{x^{2}}{2}\right)
$$

and $\varphi^{\prime}(x), \varphi^{\prime \prime}(x), \varphi^{(3)}(x)$ are $\varphi(x)$ 's $1 \rightarrow 4^{\text {th }}$ order derivatives. To any $\varphi^{(v)}(x)$, we have:

$$
\varphi^{(v)}(x)=(-1)^{v} H_{v}(x) \varphi(x)
$$

where, $H_{v}(x)$ is Hermite polynomial:

$$
H_{m}(x)=m ! \sum_{k=0}^{m / 2} \frac{(-1)^{k}}{2^{k} k !(m-2 k) !} x^{m-2 k}
$$

Therefore, to any given real stock's distribution, we can find an asymptotic way to approach it as shown above. Once we get the improved distribution function, we calculate the probability as follows in order to figure out VaR:

$$
G(A)=\int_{A}^{\infty} f(x) d x=\int_{A}^{\infty}\left[c_{0} \phi(x)+c_{1} \phi^{\prime}(x)+\frac{c_{2}}{2 !} \phi^{\prime \prime}(x)+\ldots . .\right] d x
$$

We get:

$$
G(A)=F(A)-\sum c_{n} \phi^{(n-1)}(A)
$$

with 


$$
F(A)=\int_{A}^{\infty} \phi(x) d x
$$

Start from Eq.(1.5B), we obtain:

$$
\operatorname{VaR}_{\alpha}=A=G^{-1}(\alpha) .
$$

Equation (1.1B) is the so-called Gram-Charlier expansion.

Now calculate c's:

First we have:

$$
<x^{2 N+1}>_{0}=\int_{-\infty}^{\infty} \phi(x) x^{2 N+1} d x=0
$$

and

$$
<x^{2 N}>_{0}=\int_{-\infty}^{\infty} \phi(x) x^{2 N} d x=(2 N-1) ! !
$$

For $c_{0}$ :

$$
1=\int_{-\infty}^{\infty} f(x) d x=c_{0}
$$

Where we used:

$$
\begin{aligned}
& 0=\int_{-\infty}^{\infty} \phi^{\prime}(x) d x=\int_{-\infty}^{\infty} \phi^{\prime \prime}(x) d x=. . \\
& 1=\int_{-\infty}^{\infty} \phi(x) d x
\end{aligned}
$$

Next calculate $c_{1}$ :

$$
\mu=\int_{-\infty}^{\infty} f(x) x d x=-c_{1}=\sum_{i=1}^{N} x_{i} / N
$$

Here we used:

$$
0=\int_{-\infty}^{\infty} \phi(x) x d x=\int_{-\infty}^{\infty} \phi^{\prime \prime}(x) x d x=\ldots
$$

Calculate $c_{2}$

$$
\int_{-\infty}^{\infty} f(x) x^{2} d x=c_{2}+(2-1) ! !=\sigma^{2}+\mu^{2}=\sum_{i=1}^{N} x_{i}^{2} / N
$$

so

$$
c_{2}=<x^{2}>-1
$$


Similarly

$$
\begin{aligned}
& c_{3}=-\int_{-\infty}^{\infty} f(x) x^{3} d x=-\sum_{i=1}^{N} x_{i}^{3} / N \\
& c_{4}=\sum_{i=1}^{N} x_{i}^{4} / N-3=<x^{4}>-3-6 c_{2} \\
& \ldots \ldots \ldots \ldots \\
& c_{n}=(-1)^{N} \sum_{i=1}^{N} x_{i}^{n} / N-\frac{1}{2}\left(1+(-1)^{N}\right)(N-1) ! !-\sum_{k=1}^{[N / 2]-1} \frac{N !(2 k-1) ! !}{(N-2 k) ! 2 k !} c_{N-2 k}
\end{aligned}
$$

From above computation, we obtain:

$$
\begin{aligned}
& c_{0}=1 ; \\
& c_{1}=-\mu=<x>=\sum x_{i} / N ; \\
& c_{2}=\sigma^{2}+\mu^{2}-1=\sum x_{i}^{2} / N-1 ;
\end{aligned}
$$

Therefore, the expanded distribution function is as:

$$
f(x)=\phi(x)-\mu \phi^{\prime}(x)+\frac{\sigma^{2}+\mu^{2}-1}{2 !} \phi^{\prime \prime}(x)-\frac{\gamma}{3 !} \phi^{\prime \prime \prime}(x)+\ldots
$$

where, $\gamma=\sum_{i=1}^{N} x_{i}^{3} / N=<x^{3}>$ is the first term including additional information from the data than the normal distribution, which should present the first distorted additional distribution other than the normal distribution.

\section{References}

Markowitz, Harry. (1952). Portfolio Selection, Journal of Finance, 7,79-91.

Beder, T. S. (1995). VaR: seductive but dangerous, Financial Analyst Journal, Sep-Oct, 12-24.

Engle, R. F. \& Manganelli, S. (1999). Conditional value at risk by quantile regression, NBER Working paper 7341.

Boudoukh J., M. Richardson \& R. F. Whitelaw. (1998). The best of both worlds Risk, 11:64-67.

Engle, R.F. \&V. Ng. (1993). Measuring and Testing the Impact of News On Volatility, Journal of Finance 48: 1749-1778.

Foresi, S. \& F. Peracchi. (1995), The conditional distribution of excess returns and empirical analysis, Journal of the American Statisitcal Association 90: 451-466.

Jarrow, R. \& Rudd, A. (1982).Gram-Charlier densities. Journal of Economic Dynamics \& Control 25, 1457-1483.

Parton, D.E. \& Dennis, K. E. R. (1952). The conditions under which Gram-Charlier and Edgeworth curves are positive definite and unimodal. Biometrika 39, 425-427.

Granger, C.W. J., H. White \& M. Kamstra. (1989). Interval forcasting an analysis based upon ARCH quantile estimatiors, Journal of Econometrics 40:87-96.

Hong, Y. (1999). Hypothesis Testing in Time Series via the Empirical Characteristic Function: A Generalized Spectral Density Approach, Journal of the American Statistical Association, 94, 1201-1220.

Georges A. Darbellay \& Diethelm Wuertz. (2000).The entropy as a tool for analyzing statistical dependences in financial time series, Physica A, 287, 429-439.

M.L.Mehta. (1995). Random Matrix Theory, Academic Press, New York. 
Table 1.VaR results from the corrections.

\begin{tabular}{|l|l|l|l|l|l|l|}
\hline Real data & $\begin{array}{l}\text { VAR(Normal } \\
\text { Distribution) }\end{array}$ & $\begin{array}{l}\text { VAR(1st } \\
\text { correction to } \\
\text { order L1) }\end{array}$ & $\begin{array}{l}\text { VAR(2nd } \\
\text { correction to } \\
\text { L2) }\end{array}$ & $\begin{array}{l}\text { VAR(3rd order } \\
\text { to L3) }\end{array}$ & $\begin{array}{l}\text { VAR(4th } \\
\text { order } \\
\text { to L4) }\end{array}$ & $\begin{array}{l}\text { VAR(5th } \\
\text { order to } \\
\text { L5) }\end{array}$ \\
\hline $1.48 \sigma \mathrm{N}$ & $1.65 \mathrm{~N} \sigma \mathrm{N}$ & $1.43 \mathrm{~N} \sigma \mathrm{N}$ & $1.32 \sigma \mathrm{N}$ & $0.56 \sigma \mathrm{N}$ & $1.88 \sigma \mathrm{N}$ & $1.25 \sigma \mathrm{N}$ \\
\hline
\end{tabular}

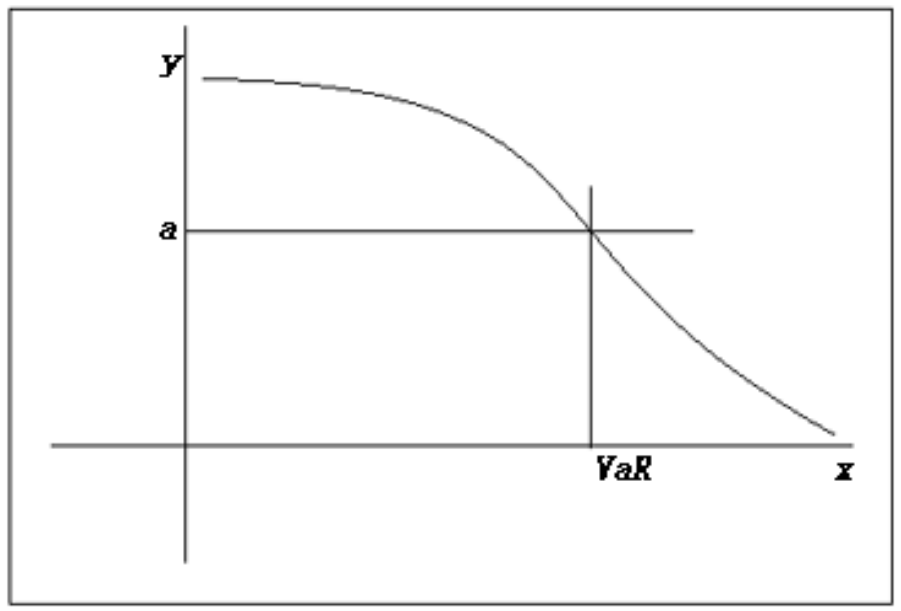

Figure 1. Schematic drawing of obtaining VaR from the modified distribution function.

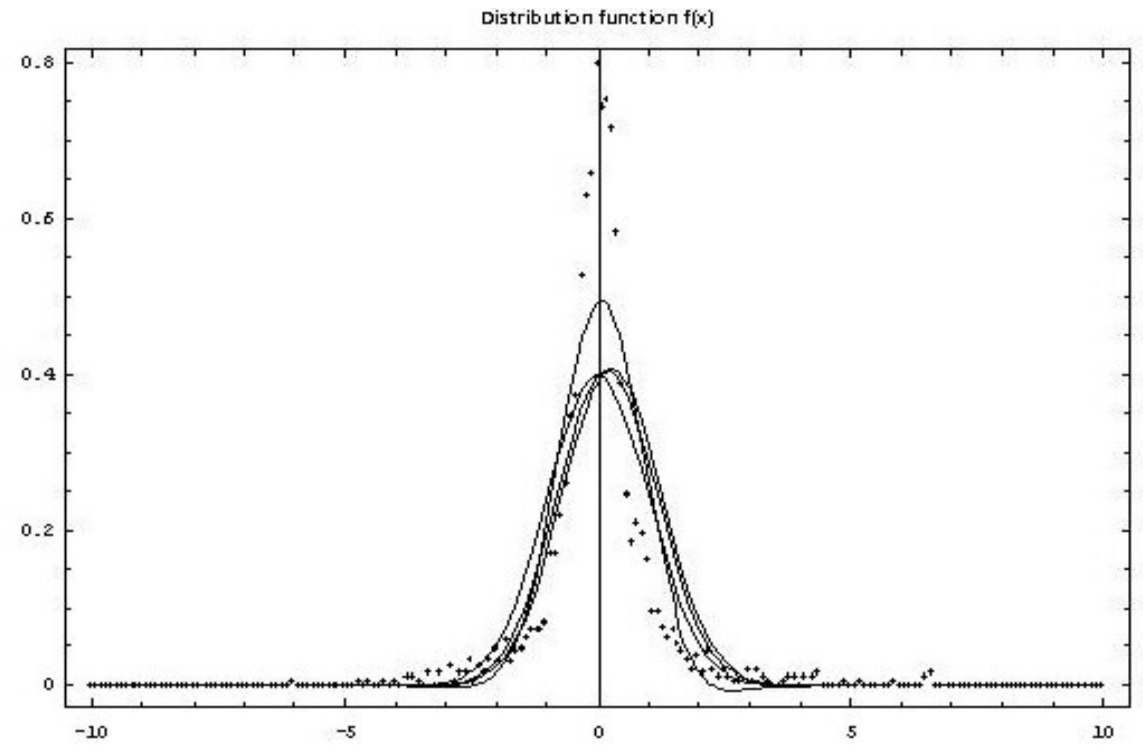

Figure 2. The distribution functions of the models:1) doted curve is the real data distribution;2) lowest curve is normal distribution;3) second lowest is the L1 correction;4) third lowest is the L2 correction;5) top curve is the L5 corrections. 